\title{
ANALYTICAL SOLUTION OF (2+1) DIMENSIONAL DIRAC EQUATION IN TIME-DEPENDENT NONCOMMUTATIVE PHASE-SPACE
}

\author{
ILYAS HAOUAM
}

\author{
Université Frères Mentouri, Laboratoire de Physique Mathématique et de Physique Subatomique (LPMPS), \\ Constantine 25000, Algeria \\ correspondence: ilyashaouam@live.fr
}

\begin{abstract}
In this article, we studied the system of a $(2+1)$ dimensional Dirac equation in a time-dependent noncommutative phase-space. More specifically, we investigated the analytical solution of the corresponding system by the Lewis-Riesenfeld invariant method based on the construction of the Lewis-Riesenfeld invariant. Knowing that we obtained the time-dependent Dirac Hamiltonian of the problem in question from a time-dependent Bopp-Shift translation, then used it to set the LewisRiesenfeld invariant operators. Thereafter, the obtained results were used to express the eigenfunctions that lead to determining the general solution of the system.
\end{abstract}

KEywords: Lewis-Riesenfeld invariant method, time-dependent Bopp-Shift translation, Bopp's Shift; time-dependent Dirac equation, time-dependent noncommutative phase-space.

\section{INTRODUCTION}

It is known that Heisenberg suggested the option of noncommutative (NC) space-time in 1930, and in 1947, Snyder presented it [1, 2] to the necessity of regularizing the divergence of the quantum field theory. Then, in recent years, noncommutative geometry (NCG) became very interesting for studying several physical problems, and it became clear that there is a strong connection between the NCG and string theories. Studies of this geometric type and its involvement have been incorporated with important physical concepts and tools, and have been useful for highlighting in various fields of physics, particularly in matrix theory (matrix model BFSS (1997)) [3]. The NCG was involved also in the description of quantum gravity theories [4], Aharonov-Bohm effect [5], Aharonov-Casher effect [6], etc [7]. Knowing that the origins of the NCG related to the investigations for topological spaces of $\mathrm{C} *$-algebras of functions. Later this type of geometry was theorized by A. Connes and others in 1985 [8-12] by studying and defining a cyclical cohomology. It has been shown that the differential calculus on manifolds had an NC equivalent. Next, the NCG found great encouragement through several mathematical results, such as the K-theory of $\mathrm{C} *$-algebras, Gelfand-Naïmark theorem on the $\mathrm{C} *$-algebras, characterizations of commutative von Neumann algebras, cyclic cohomology of the $C^{\infty}(M)$ algebra, relations between Dirac operators and Riemannian metrics, Serre-Swan theorem, etc. The idea of the phase-space noncommutativity is largely motivated by the foundations of quantum mechanics through the canonical quantization.

It is easy to apply the phase-space noncommutativity using the ordinary product with Weyl operators (Weyl-Wigner maps) [12, or by replacing the ordinary product with the Moyal-Weyl product ( $\star$-product) in the functions and actions of our systems [13, 14, also the Bopp-shift linear transformations [15, 16], and the Seiberg-Witten maps [8, 10, 17].

Studying physics within the NCG has attracted a lot of interest in recent years, because noncommutativity is necessary when considering the low-energy efficiency of D-brane with a background magnetic field, and also in a tiny scale of strings or in conditions of a very high energy, the effects of noncommutativity may appear. Besides, one of the strong motivations of the NCG, is to obtain a coherent mathematical framework in which it would be possible to describe quantum gravitation. For all these reasons and advantages, we carry out this work in the NC formalism. In addition, it is interesting to find other models in which noncommutativity emerges.

Several scientific works have focused on the time-independent noncommutativity. Experimental research has considered the parameters of the noncommutativity of fixed values in the context of cosmic microwave background radiation, perhaps considered approximately fixed to the celestial sphere, for example, as proposed in ref [18. However, differently, in our work, our obvious intention is to involve the time-dependency in the NC parameters because of the possibility that NC parameters may show time-dependency. For instance, physical measurements must take into account the effect of the Earth's rotation around its axis, which causes a time-dependency in the NC parameters.

However, the motivations for choosing to study the $(2+1)$ dimensional Dirac equation are due to several important works in this context, such as the investigations of Landau levels [19, the oscillation of magnetization 
[20], Weiss oscillation [21], de Haas-van Alphen effect [22], analysis through coherent states [23], the movement of electrons transporters in graphene and other materials [24, etc. Particularly, the 2 dimensional Dirac equation in interaction with a homogeneous magnetic field has various applications in graphene, such as in refs [25, 26], and in studying quantum Hall effect and fractional Hall effects [27, 28, Berry phase [29], etc. In graphene and other materials such as in Weyl semimetals, an important phenomenon takes place if the magnetic field and the uniform electric field are introduced. Specifically, the spacing between different Landau levels decreases if the electric field strength reaches a critical value [30, 31].

In our study about a $(2+1)$ dimensional Dirac system, the noncommutativity will be considered timedependent through a time-dependent Darboux transformation "Bopp's shift". This, in turn, makes the studied system a time-dependent one, $\mathcal{H}\left(x_{i}^{n c}, p_{i}^{n c}\right) \longrightarrow \mathcal{H}(t)$.

Solving the system of equations in interaction with time-dependent potentials has attracted many physicists over the past years. In addition to the essential mathematical benefit, this topic is related to a lot of physical problems and applications for its applicability. For instance, in quantum transport [32 34], quantum optics [35 37], quantum information [38, the degenerate parametric amplifier [39], also spintronics [40, 41], and in the description of the two trapped cold ions dynamics in the Paul trap 42 . To study systems of time-dependent equations, there are many methods like the evolution operator, the change of representation, the unitary transformations, path integral, second quantization, Lewis-Riesenfeld (LR) invariant approach. Also, there are other used techniques, as in refs [43, 44].

The LR method [45, 46] is a technique that allows obtaining a set of solutions of time-dependent equation systems, through the eigenstates of LR invariants. These invariants are built to find the solutions of such systems of equations, where Lewis and Riesenfeld in their original paper presented a technique to obtaining a group of exact wave-functions for the time-dependent harmonic oscillator in Hilbert space. The LR approach has been applied in many applications such as in mesoscopic $\mathrm{R}(\mathrm{t}) \mathrm{L}(\mathrm{t}) \mathrm{C}(\mathrm{t})$ electric circuits where the quantum evolution is described [47]. As well as in engineering, in shortcuts and adiabaticity [48]...

A large variety of scientific papers concerning time-dependent systems were interested in the time-dependent harmonic oscillator, or in time-dependent linear potentials, but in our current work, to be more specific, we report the time-dependent Background of the NC phase-space. We consider a time-dependent Bopp-shift translation to transform the system to a time-dependent $\mathrm{NC}$ one, then, due to the LR invariant method, we obtain the LR invariant and its eigenstates to solve our system equations.

\section{TIME-DEPENDENT NONCOMMUTATIVITY}

In theory, the NCG space may not commute anymore (i.e. $\mathrm{AB} \neq \mathrm{BA}$ ). In a $d$ dimensional time-dependent $\mathrm{NC}$ phase-space, let us consider the operators of coordinates and momentum $x_{j}^{n c}$ and $p_{k}^{n c}$, respectively. These NC operators satisfy the deformed commutation relations [49]

$$
\begin{aligned}
{\left[x_{j}^{n c}, x_{k}^{n c}\right] } & =i \Theta_{j k}(t) \\
{\left[p_{j}^{n c}, p_{k}^{n c}\right] } & =i \eta_{j k}(t), \quad(j, k=1, . ., d) \\
{\left[x_{j}^{n c}, p_{k}^{n c}\right] } & =i \hbar^{e f f} \delta_{j k}
\end{aligned}
$$

the effective Planck constant being

$$
\hbar^{e f f}=\hbar\left(1+\frac{\Theta \eta}{4 \hbar^{2}}\right)
$$

where $\frac{\Theta \eta}{4 \hbar^{2}} \ll 1$ is the consistency condition in the usual quantum mechanics. $\delta_{j k}$ is the identity matrix, and $\Theta_{j k}, \eta_{j k}$ are real constant antisymmetric $d \times d$ matrices.

In some studies concerning the NC parameters, as in the experiment by "Nesvizhevsky et al" [50, 51], we note that $\Theta \approx 10^{-30} \mathrm{~m}^{2}$ and $\eta \approx 1,76 \cdot 10^{-61} \mathrm{Kg}^{2} \mathrm{~m}^{2} \mathrm{~s}^{-2}$. Other bounds exist. For example, $\Theta \approx 4.10^{-40} \mathrm{~m}^{2}$ when assuming the natural units, $\hbar=c=1$ [52]. As well as when taking into account that the experimental energy resolution is related to the uncertainty principle because of the finite lifetime of the neutron, this leads to obtaining $\eta \approx 10^{-67} \mathrm{Kg}^{2} \mathrm{~m}^{2} \mathrm{~s}^{-2}$ (a kind of correction). These obtained results including the experiment by "Nesvizhevsky et al", allow us to evaluate the consistency condition of the NC model $\left|\frac{\Theta \eta}{4 \hbar^{2}}\right| \preceq 10^{-24}$. But if we consider the modifications introduced by noncommutativity over $\hbar$ value (the precision is about $10^{-9}$ ), which are at least about 24 orders of magnitude smaller than its value, with considering the corrected bounds of $\eta$, we have $\left|\frac{\Theta \eta}{4 \hbar^{2}}\right| \preceq 10^{-29}$ [53]. These values agree with the higher limits on the basic scales of coordinate and momentum. These limits will be suppressed if the used magnetic field in the experiment is weak, about $B \approx 5 m G$.

As long as the system in which we investigate the effects of noncommutativity is 2 dimensional, we restrict 
ourselves to the following $\mathrm{NC}$ algebra

$$
\begin{aligned}
{\left[x_{j}^{n c}, x_{k}^{n c}\right] } & =i \Theta e^{\gamma t} \epsilon_{j k} \\
{\left[p_{j}^{n c}, p_{k}^{n c}\right] } & =i \eta e^{-\gamma t} \epsilon_{j k}, \quad(j, k=1,2) \\
{\left[x_{j}^{n c}, p_{k}^{n c}\right] } & =i \hbar^{e f f} \delta_{j k}
\end{aligned}
$$

we have $\epsilon_{12}=-\epsilon_{21}=1, \epsilon_{11}=\epsilon_{22}=0$, and $\Theta, \eta$ are real-valued with the dimension of length ${ }^{2}$ and momentum ${ }^{2}$, respectively.

While the space coordinates and momentum are fuzzy and fluid [54, they cannot be localized, unless for minus infinite times. The parameters $\Theta, \eta$ represent the fuzziness and $\gamma$ represents the fluidity of the space. The above equation is the relation of the ordinary NCG except that the NC structure constants are considered as exponentially increasing functions with the evolution of time. Certainly, there is a multitude of other possibilities, such as $\Theta(t)=\Theta \cos (\gamma t), \eta(t)=\eta \sin (\gamma t)$.

The new deformed geometry can be described by the operators

$$
\begin{array}{ll}
x_{1}^{n c}=x^{n c}=x-\frac{1}{2 \hbar} \Theta e^{\gamma t} p_{y}, & p_{1}^{n c}=p_{x}^{n c}=p_{x}+\frac{1}{2 \hbar} \eta e^{-\gamma t} y \\
x_{2}^{n c}=y^{n c}=y+\frac{1}{2 \hbar} \Theta e^{\gamma t} p_{x}, & p_{2}^{n c}=p_{y}^{n c}=p_{y}-\frac{1}{2 \hbar} \eta e^{-\gamma t} x
\end{array} .
$$

When $\gamma=0$, the time-dependency in the structure of NC parameters vanishes. In addition, for $\Theta=\eta=0$, the NCG reduces to a commutative one and the coordinates $x_{j}$ and the momentum $p_{k}$ satisfy the ordinary canonical commutation relations

$$
\begin{aligned}
& {\left[x_{j}, x_{k}\right]=0} \\
& {\left[p_{j}, p_{k}\right]=0, \quad(j, k=1,2) .}
\end{aligned}
$$

\section{3. $(2+1)$ D EXPlicitly time-DEPEndent DiraC EQUATION AND its invariant operator}

\section{1. $(2+1)$ D DiRAC EQUATION IN TIME-DEPENDENT NONCOMMUTATIVE PHASE-SPACE}

In presence of an electromagnetic four-potential $A_{\mu}=\left(A_{0}, A_{i}\right)$, the Dirac equation in $(2+1) \mathrm{d}$ is given by

$$
\left(c \alpha_{i}\left(p_{i}-\frac{e}{c} A_{i}(x)\right)+e A_{0}(x)+\beta m c^{2}\right)|\psi\rangle=i \hbar \frac{\partial}{\partial t}|\psi\rangle,
$$

with $|\psi\rangle$ being the Dirac wave function, and $p_{j}=\left(\begin{array}{c}p_{x} \\ p_{y} \\ p_{z}\end{array}\right)$ is the momentum. The Dirac matrices $\alpha_{j}, \beta$

$$
\alpha_{j}=\left(\begin{array}{cc}
0 & \sigma_{j} \\
\sigma_{j} & 0
\end{array}\right), \alpha_{1}=\sigma_{1}=\left(\begin{array}{cc}
0 & 1 \\
1 & 0
\end{array}\right), \alpha_{2}=\sigma_{2}=\left(\begin{array}{cc}
0 & -i \\
i & 0
\end{array}\right), \beta=\sigma_{3}=\left(\begin{array}{cc}
1 & 0 \\
0 & -1
\end{array}\right), \mathcal{I}=\left(\begin{array}{ll}
1 & 0 \\
0 & 1
\end{array}\right),
$$

satisfy the following anticommutation relations

$$
\left\{\alpha_{i}, \alpha_{j}\right\}=2 \delta_{i j},\left\{\alpha_{i}, \beta\right\}=0 \text { with } \alpha_{i}^{2}=\beta^{2}=1 .
$$

We consider the magnetic field $\vec{B}$ along z-direction, and it is defined in terms of the symmetric potential

$$
A_{i}=\frac{B}{2}(-y, x, 0), \text { with } A_{0}=0,
$$

most research about time-dependent systems concerns the presence of an electric field. However, in our current work, we do not rely on the electric field.

Using Eq.99, the Hamiltonian of the system becomes

$$
\mathcal{H}\left(x, y, p_{x} p_{y}\right)=c \alpha_{1} p_{x}+c \alpha_{2} p_{y}+e \alpha_{1} \frac{B}{2} y-e \alpha_{2} \frac{B}{2} x+\beta m c^{2} .
$$

Achieving the NCG in the Dirac Hamiltonian (10) as follows

$$
\mathcal{H}\left(x^{n c}, y^{n c}, p_{x}^{n c}, p_{y}^{n c}\right)=c \alpha_{1} p_{x}^{n c}+c \alpha_{2} p_{y}^{n c}-e \alpha_{2} \frac{B}{2} x^{n c}+e \alpha_{1} \frac{B}{2} y^{n c}+\beta m c^{2},
$$

by applying Eq.44, we necessarily express the new NC Hamiltonian using the commutative variables $\left\{x, y, p_{x}, p_{y}\right\}$, and by assuming that $\hbar=c=1$ (natural units) to simplify the calculations, then we obtain

$$
\mathcal{H}^{n c}\left(x, y, p_{x} p_{y}, t\right)=\alpha_{1}\left(1+\frac{e B}{4} \Theta e^{\gamma t}\right) p_{x}-\alpha_{2}\left(\frac{e B}{2}+\frac{\eta}{2} e^{-\gamma t}\right) x+\alpha_{2}\left(1+\frac{e B}{4} \Theta e^{\gamma t}\right) p_{y}+\alpha_{1}\left(\frac{e B}{2}+\frac{\eta}{2} e^{-\gamma t}\right) y+\beta m
$$


The time-dependent Dirac equation in NC phase-space is given by

$$
i \frac{\partial}{\partial t}|\bar{\psi}(t)\rangle=\mathcal{H}^{n c}(t)|\bar{\psi}(t)\rangle
$$

where $|\bar{\psi}(t)\rangle$ is the Dirac NC wave function.

\subsection{The COnstruction of the LeWis-Riesenfeld InVARIANTS}

To solve Eq. 13), we use the LR invariant method, which assumes the existence of a quantum-mechanical invariant $\mathrm{I}(\mathrm{t})$ which satisfies

$$
\frac{d I(t)}{d t}=-i\left[I(t), \mathcal{H}^{n c}(t)\right]+\frac{\partial I(t)}{\partial t}=0
$$

with

$$
i \frac{\partial}{\partial t}(I(t)|\bar{\psi}(t)\rangle)=\mathcal{H}^{n c}(t) I(t)|\bar{\psi}(t)\rangle .
$$

The Eq.(14) is called the invariance condition for the dynamical invariant operator $I(t)$, which is a Hermitian operator

$$
I(t)=I^{+}(t)
$$

Assuming that

$$
I(t)=A_{1}(t) p_{x}+B_{1}(t) x+A_{2}(t) p_{y}+B_{2}(t) y+C(t),
$$

with $A_{1}(t), B_{1}(t), A_{2}(t), B_{2}(t), C(t)$ are time-dependent matrices. The substitution of Eqs. 17. 11 into Eq. 14. and using the properties of the commutation relations lead to

$$
\left[I, \mathcal{H}^{n c}\right]+i \frac{\partial I}{\partial t}=\left[A_{1} p_{x}, \mathcal{H}^{n c}\right]+\left[B_{1} x, \mathcal{H}^{n c}\right]+\left[A_{2} p_{y}, \mathcal{H}^{n c}\right]+\left[B_{2} y, \mathcal{H}^{n c}\right]+\left[C, \mathcal{H}^{n c}\right]+i \frac{\partial I}{\partial t}=0
$$

for simplicity, we take $f_{\Theta}(t)=1+\frac{e B}{4} \Theta e^{\gamma t}$ and $f_{\eta}(t)=\frac{e B}{2}+\frac{\eta}{2} e^{-\gamma t}$, which are not matrices, then we have

$$
\begin{gathered}
\quad\left[A_{1}, \alpha_{1} f_{\Theta}\right] p_{x}^{2}+\left[A_{2}, \alpha_{2} f_{\Theta}\right] p_{y}^{2}-\left[B_{1}, \alpha_{2} f_{\eta}\right] x^{2}+\left[B_{2}, \alpha_{1} f_{\eta}\right] y^{2}+\left\{\left[A_{1}, \alpha_{2} f_{\Theta}\right]+\left[A_{2}, \alpha_{1} f_{\Theta}\right]\right\} p_{x} p_{y} \\
+\left\{\left[A_{1}, \alpha_{1} f_{\eta}\right]+\left[B_{2}, \alpha_{1} f_{\Theta}\right]\right\} y p_{x}+\left\{\left[A_{1}, \beta m\right]+\left[C, \alpha_{1} f_{\Theta}\right]+i \frac{\partial A_{1}}{\partial t}\right\} p_{x}+\left\{\left[A_{2}, \beta m\right]+\left[C, \alpha_{2} f_{\Theta}\right]+\frac{\partial A_{2}}{\partial t}\right\} p_{y} \\
+\left\{\left[B_{1}, \beta m\right]-\left[C, \alpha_{2} f_{\eta}\right]+i \frac{\partial B_{1}}{\partial t}\right\} x+\left\{\left[B_{2}, \beta m\right]+\left[C, \alpha_{1} f_{\eta}\right]+i \frac{\partial B_{2}}{\partial t}\right\} y+\left\{-\left[A_{1}, \alpha_{2} f_{\eta}\right]+\left[B_{1}, \alpha_{1} f_{\Theta}\right]\right\} x p_{x} \\
\quad+\left\{\left[B_{1}, \alpha_{2} f_{\Theta}\right]-\left[A_{2}, \alpha_{2} f_{\eta}\right]\right\} x p_{y}+\left\{\left[B_{1}, \alpha_{1} f_{\eta}\right]-\left[B_{2}, \alpha_{2} f_{\eta}\right]\right\} x y+\left\{\left[A_{2}, \alpha_{1} f_{\eta}\right]+\left[B_{2}, \alpha_{2} f_{\Theta}\right]\right\} y p_{y} \\
\quad+i A_{1} \alpha_{2} f_{\eta}+i B_{1} \alpha_{1} f_{\Theta}-i A_{2} \alpha_{1} f_{\eta}+i B_{2} \alpha_{2} f_{\Theta}-i\left[B_{1}, \alpha_{1} f_{\Theta}\right]-i\left[B_{2}, \alpha_{2} f_{\Theta}\right]+[C, \beta m]+i \frac{\partial C}{\partial t}=0
\end{gathered}
$$

Then, to satisfy Eq.(14), and always taking advantage of the properties of commutation relations, with $p_{i} p_{j}=p_{j} p_{i}, x_{i} p_{j}=p_{j} x_{i}$ if $\mathrm{i} \neq \mathrm{j} \in\{1,2\}$, else $p_{x} x=x p_{x}-i, p_{y} y=y p_{y}-i$. We demand

$$
\begin{gathered}
{\left[A_{1}, \alpha_{1} f_{\Theta}\right]=0,} \\
{\left[A_{2}, \alpha_{2} f_{\Theta}\right]=0,} \\
{\left[B_{1}, \alpha_{2} f_{\eta}\right]=0,} \\
{\left[B_{2}, \alpha_{1} f_{\eta}\right]=0,} \\
{\left[A_{1}, \beta\right] m+\left[C, \alpha_{1} f_{\Theta}\right]+i \frac{\partial A_{1}}{\partial t}=0,} \\
{\left[A_{2}, \beta\right] m+\left[C, \alpha_{2} f_{\Theta}\right]+i \frac{\partial A_{2}}{\partial t}=0,} \\
{\left[B_{1}, \beta\right] m-\left[C, \alpha_{2} f_{\eta}\right]+i \frac{\partial B_{1}}{\partial t}=0,} \\
{\left[B_{2}, \beta\right] m+\left[C, \alpha_{1} f_{\eta}\right]+i \frac{\partial B_{2}}{\partial t}=0,} \\
{\left[A_{1}, \alpha_{2} f_{\Theta}\right]+\left[A_{2}, \alpha_{1} f_{\Theta}\right]=0,} \\
{\left[B_{1}, \alpha_{1} f_{\Theta}\right]-\left[A_{1}, \alpha_{2} f_{\eta}\right]=0,} \\
{\left[B_{1}, \alpha_{2} f_{\Theta}\right]-\left[A_{2}, \alpha_{2} f_{\eta}\right]=0,} \\
{\left[B_{1}, \alpha_{1} f_{\eta}\right]-\left[B_{2}, \alpha_{2} f_{\eta}\right]=0,} \\
{\left[B_{2}, \alpha_{1} f_{\Theta}\right]+\left[A_{1}, \alpha_{1} f_{\eta}\right]=0,}
\end{gathered}
$$




$$
\begin{gathered}
{\left[A_{2}, \alpha_{1} f_{\eta}\right]+\left[B_{2}, \alpha_{2} f_{\Theta}\right]=0,} \\
i A_{1} \alpha_{2} f_{\eta}+i B_{1} \alpha_{1} f_{\Theta}-i A_{2} \alpha_{1} f_{\eta}+i B_{2} \alpha_{2} f_{\Theta}-i\left\{\left[B_{1}, \alpha_{1} f_{\Theta}\right]+\left[B_{2}, \alpha_{2} f_{\Theta}\right]\right\}+[C, \beta m]+i \frac{\partial C}{\partial t}=0 .
\end{gathered}
$$

From the relations $(20-23)$, and as long as from Eq. 20$]$, we have

$$
A_{1}=a_{0}(t)+a_{1}(t) \alpha_{1}+a_{2}(t) \alpha_{1}^{2}+a_{3}(t) \alpha_{1}^{3}+a_{4}(t) \alpha_{1}^{4}+\ldots=a_{0}^{\prime}(t)+a_{1}^{\prime}(t) \alpha_{1}, \text { with } a_{i}^{\prime}(t)=a_{i}(t),
$$

therefore, we obtain

$$
\begin{aligned}
& A_{1}=a_{1}+a_{2} \alpha_{1}, \\
& A_{2}=a_{3}+a_{4} \alpha_{2}, \\
& B_{1}=b_{1}+b_{2} \alpha_{2}, \\
& B_{2}=b_{3}+b_{4} \alpha_{1} .
\end{aligned}
$$

From Eqs. 24- 27) and with the same manner, supposing that $C$ is written in terms of $\alpha_{1}, \alpha_{2}$ and $\beta$ as follows

$$
C=c_{1}+c_{2} \alpha_{1}+c_{3} \alpha_{2}+c_{4} \beta,
$$

where $a_{j}, b_{j}$ and $\mathrm{c}_{j}$ (with $j=1, . ., 4$ ) are supposed to be time-dependent arbitrary functions. Substituting Eqs. (40, 36) into Eq.24) and Eqs.440, 37) into Eq.25), and taking into consideration Eq.(8), yields

$$
\frac{\partial a_{1}}{\partial t}=0, \quad \frac{\partial a_{3}}{\partial t}=0, \quad a_{2}=a_{4}=c_{2}=c_{3}=c_{4}=0,
$$

thereafter, substituting Eqs.40, 38 into Eq.267 and Eqs.40, 39) into Eq.27), and taking into consideration Eq. (8), yields

$$
\frac{\partial b_{1}}{\partial t}=0, \quad \frac{\partial b_{3}}{\partial t}=0, \quad b_{2}=b_{4}=c_{2}=c_{4}=0 .
$$

From the Eqs.41, 42 we note that $a_{1}, a_{3}, b_{1}, b_{3}$ are time-independent constants. We have

$$
A_{1}=a_{1}, \quad A_{2}=a_{3}, \quad B_{1}=b_{1}, \quad B_{2}=b_{3}, \quad C=c_{1} .
$$

In addition, from Eqs. 30, 32, and assuming that there exist $\chi(t)$ and $\varphi(t)$, which are time-dependent matrices, with $\left[\chi(t), \alpha_{2}\right]=\left[\varphi(t), \alpha_{1}\right]=0$. The time-dependency may appear as follows

$$
\begin{aligned}
& b_{1} f_{\Theta}-a_{3} f_{\eta}=\chi(t) \\
& b_{3} f_{\Theta}+a_{1} f_{\eta}=\varphi(t)
\end{aligned} .
$$

Now, substituting Eq.443) into Eq.(34) and using Eq.444) gives us

$$
\frac{\partial c_{1}}{\partial t}=-\left\{a_{1} f_{\eta}+b_{3} f_{\Theta}\right\} \alpha_{2}-\left\{b_{1} f_{\Theta}-a_{3} f_{\eta}\right\} \alpha_{1},
$$

using system of relations [44], we find

$$
\frac{\partial c_{1}}{\partial t}=0 \text { and } \chi=\varphi=0 .
$$

Last but not least, the dynamical invariant (17) of the time-dependent NC Dirac equation can be written as follows

$$
I=a_{1} p_{x}+b_{1} x+a_{3} p_{y}+b_{3} y+c_{1},
$$

we inferred that Eq.(14) is verified and $c_{1}$ should be a constant. We may also note that all the spin-dependent parts, which are proportional to $\alpha_{j}, \beta$ disappear. Which means that $I$ has no spin-dependency, but it is proportional to the matrix of identity in the spinor of space. 


\subsection{Eigenvalues and eigenstates of $I$ and $\mathcal{H}(t)$}

Supposing that the invariant in general $I(t)$ is a complete set of eigenfunctions $|\phi(\lambda, k)\rangle$ (in this subsection, the analysis is not concerning only on time-independent invariants), with $\lambda$ being the corresponding eigenvalue (spectrum of the operator), and $k$ represents all other necessary quantum numbers to specify the eigenstates, the eigenvalues equation is written as

$$
I(t)|\phi(\lambda, k)\rangle=\lambda|\phi(\lambda, k)\rangle,
$$

where $|\phi(\lambda, k)\rangle$ are an orthogonal eigenfunctions

$$
\left\langle\phi(\lambda, k) \mid \phi\left(\lambda^{\prime}, k^{\prime}\right)\right\rangle=\delta_{\lambda \lambda^{\prime}} \delta_{k k^{\prime}} .
$$

According to Eq. (16), the eigenvalues are real and not time-dependent. Deriving Eq.(48) in time, we find

$$
\frac{\partial I}{\partial t}|\phi(\lambda, k)\rangle+I \frac{\partial}{\partial t}|\phi(\lambda, k)\rangle=\frac{\partial \lambda}{\partial t}|\phi(\lambda, k)\rangle+\lambda \frac{\partial}{\partial t}|\phi(\lambda, k)\rangle,
$$

we apply Eq.(14) over the eigenfunctions $|\phi(\lambda, k)\rangle$, we have

$$
i \frac{\partial I}{\partial t}|\phi(\lambda, k)\rangle+I \mathcal{H}^{n c}|\phi(\lambda, k)\rangle-\mathcal{H}^{n c} \lambda|\phi(\lambda, k)\rangle=0,
$$

the scalar product of Eq. 51 by $\left\langle\phi\left(\lambda^{\prime}, k^{\prime}\right)\right|$ is

$$
i\left\langle\phi\left(\lambda^{\prime}, k^{\prime}\right)\left|\frac{\partial I}{\partial t}\right| \phi(\lambda, k)\right\rangle+\left(\lambda^{\prime}-\lambda\right)\left\langle\phi\left(\lambda^{\prime}, k^{\prime}\right)\left|\mathcal{H}^{n c}\right| \phi(\lambda, k)\right\rangle=0,
$$

which implies

$$
\left\langle\phi\left(\lambda^{\prime}, k^{\prime}\right)\left|\frac{\partial I}{\partial t}\right| \phi(\lambda, k)\right\rangle=0,
$$

the scalar product of Eq. 50 by $\left\langle\phi\left(\lambda^{\prime}, k^{\prime}\right)\right|$ is

$$
\left\langle\phi\left(\lambda^{\prime}, k^{\prime}\right)\left|\frac{\partial I}{\partial t}\right| \phi(\lambda, k)\right\rangle=\frac{\partial \lambda}{\partial t}
$$

from Eq.(53), the Eq.(54) shows that

$$
\left\langle\phi\left(\lambda^{\prime}, k^{\prime}\right)\left|\frac{\partial I}{\partial t}\right| \phi(\lambda, k)\right\rangle=\frac{\partial \lambda}{\partial t}=0 .
$$

While the eigenvalues are time-independent, the eigenstates should be time-dependent.

In order to find the link between the eigenstates of the invariant $I(t)$ and the solutions of the relativistic Dirac equation, we first start with writing the motion equation of $|\phi(\lambda, k)\rangle$, so that, using Eq. 50] and Eq. 555, we obtain

$$
\frac{\partial I}{\partial t}|\phi(\lambda, k)\rangle=(\lambda-I) \frac{\partial}{\partial t}|\phi(\lambda, k)\rangle
$$

by using the scalar product with $\left\langle\phi\left(\lambda^{\prime}, k^{\prime}\right)\right|$, and taking Eq. 52$\}$ to eliminate $\left\langle\phi\left(\lambda^{\prime}, k^{\prime}\right)\left|\frac{\partial I}{\partial t}\right| \phi(\lambda, k)\right\rangle$, then we obtain

$$
i\left\langle\phi\left(\lambda^{\prime}, k^{\prime}\right)\left|\left(\lambda-\lambda^{\prime}\right) \frac{\partial}{\partial t}\right| \phi(\lambda, k)\right\rangle=\left(\lambda-\lambda^{\prime}\right)\left\langle\phi\left(\lambda^{\prime}, k^{\prime}\right)\left|\mathcal{H}^{n c}\right| \phi(\lambda, k)\right\rangle,
$$

for $\lambda^{\prime} \neq \lambda$, we deduce

$$
i\left\langle\phi\left(\lambda^{\prime}, k^{\prime}\right)\left|\frac{\partial}{\partial t}\right| \phi(\lambda, k)\right\rangle=\left\langle\phi\left(\lambda^{\prime}, k^{\prime}\right)\left|\mathcal{H}^{n c}\right| \phi(\lambda, k)\right\rangle,
$$

then we deduce immediately that $|\phi(\lambda, k)\rangle$ satisfy the Dirac equation, that is to say $|\phi(\lambda, k)\rangle$ are particular solutions of the Dirac equation.

It is assumed that a phase has been taken, but it is still always possible to multiply it by an arbitrary time-dependent phase factor, which means that we can define a new set of $I(t)$ eigenstates linked to our overall by a time-dependent gauge transformation, and

$$
|\phi(\lambda, k)\rangle_{\alpha}=e^{i \alpha_{\lambda}(t)}|\phi(\lambda, k)\rangle,
$$


where $\alpha_{\lambda}(t)$ is a real time-dependent function arbitrarily chosen called LR phase, $\left|\phi_{\lambda}(x, y, t)\right\rangle_{\alpha}$ are eigenstates of $I(t)$ which are orthonormal and associated with $\lambda$. By putting Eq. (59) in Eq. (58) and using Eq. (49), we find

$$
\frac{\partial \alpha_{\lambda, k}}{\partial t} \delta_{\lambda \lambda^{\prime}} \delta_{k k^{\prime}}=\left\langle\phi\left(\lambda^{\prime}, k^{\prime}\right)\left|i \frac{\partial}{\partial t}-\mathcal{H}^{n c}\right| \phi(\lambda, k)\right\rangle
$$

All the eigenstates of the invariant are also solutions of the time-dependent Dirac equation, it was shown in [46] that its general solution is done by

$$
|\bar{\psi}(t)\rangle=\sum_{\lambda, k} C_{\lambda, k} e^{i \alpha_{\lambda, k}(t)}|\phi(\lambda, k, t)\rangle
$$

we remark that Eq. (61) is also spin-independent in its state. But maybe the spin-dependent part is entangled in the coefficient $C .|\phi(\lambda, k, t)\rangle$ are the orthonormal eigenstates of $I(t)$, with $C_{\lambda, k}$ being time-independent coefficients, which correspond to $|\psi(0)\rangle$

$$
C_{\lambda, k}=\langle\lambda, k \mid \psi(0)\rangle
$$

For a discrete spectrum of $I(t)$, with $\lambda=\lambda^{\prime}, k=k^{\prime}$, and from Eq. 60 the LR phase is defined as

$$
\alpha(t)=\int_{0}^{t}\left\langle\phi\left(\lambda, k, t^{\prime}\right)\left|i \frac{\partial}{\partial t^{\prime}}-\mathcal{H}^{n c}\left(t^{\prime}\right)\right| \phi\left(\lambda, k, t^{\prime}\right)\right\rangle d t^{\prime}
$$

But in the continuous spectrum case, the general expression of the phase is

$$
\frac{\partial \alpha_{\lambda, k}}{\partial t}\left\langle\phi \left(\lambda^{\prime}, k^{\prime}, t^{\prime}|\phi(\lambda, k, t)\rangle=\left\langle\phi\left(\lambda^{\prime}, k^{\prime}, t^{\prime}\right)\left|i \frac{\partial}{\partial t}-\mathcal{H}^{n c}\right| \phi(\lambda, k, t)\right\rangle,\right.\right.
$$

where $k$ is an index that varies continuously in the real values, thus

$$
\left\langle\phi \left(\lambda^{\prime}, k^{\prime}, t^{\prime}|\phi(\lambda, k, t)\rangle=\delta_{\lambda \lambda^{\prime}} \delta\left(k-k^{\prime}\right)\right.\right.
$$

substituting Eq. 65) in Eq. (64) yields

$$
\alpha(t)=\iint_{0}^{t}\left\langle\phi\left(\lambda, k^{\prime}, t^{\prime}\right)\left|i \frac{\partial}{\partial t^{\prime}}-\mathcal{H}^{n c}\right| \phi\left(\lambda, k, t^{\prime}\right)\right\rangle d t^{\prime} d k^{\prime} .
$$

Once the expression of the phase $\alpha(t)$ is found, we can write the particular solution of our NC time-dependent Dirac equation 61.

For simplicity, we use the notation of the discrete spectrum of $I(t)$. We see that the eigenfunction of $I(t)$ has the form of 55,56

$$
\left|\phi_{\lambda, k}(x, y, t)\right\rangle \propto|\lambda, k\rangle \exp \left[i\left(\xi_{1}(t) x+\xi_{2}(t) y+\xi_{3}(t) x^{2}+\xi_{4}(t) y^{2}\right)\right],
$$

where $\xi_{1}(t), \xi_{2}(t), \xi_{3}(t), \xi_{4}(t)$ are arbitrary time-dependent functions.

By substituting Eq. 67) into Eq. 63) yields

$$
\alpha(t)=\vartheta-\int_{0}^{t} E^{n c} d t^{\prime}
$$

with

$$
\vartheta(x, y, t)=\left(\xi_{1}(0)-\xi_{1}(t)\right) x+\left(\xi_{2}(0)-\xi_{2}(t)\right) y+\left(\xi_{3}(0)-\xi_{3}(t)\right) x^{2}+\left(\xi_{4}(0)-\xi_{4}(t)\right) y^{2},
$$

and $E^{n c}$ is the eigenvalue of the Hamiltonian (12).

Finally, the solution of the NC Dirac equation 13 is 46.

$$
|\bar{\psi}(t)\rangle=\sum_{\lambda, k} C_{\lambda, k} e^{i\left[\vartheta-\int_{0}^{t} E^{n c} d t^{\prime}\right]}|\phi(\lambda, k, t)\rangle,
$$




\subsection{The EXACT FORM OF THE SOLUtions OF THE PROBlem}

As agreed [55 57, the wave function of the NC Dirac equation is given by the following trial function

$$
|\bar{\psi}(x, y, t)\rangle=\mathcal{F}(t) \mid \phi(x, y, t\rangle
$$

where $\mathcal{F}$ is a time-dependent vector of 2 components $(2 \times 1)$

$$
\mathcal{F}(t)=\left(\begin{array}{c}
\mathcal{F}_{1}(t) \\
\mathcal{F}_{2}(t)
\end{array}\right)
$$

as long as $I(t)$ is independent in time, Eq.15 goes to Eq. 13. Then the substitution of Eq. (71) into Eq. 13. and using Eqs. 67, 7) give

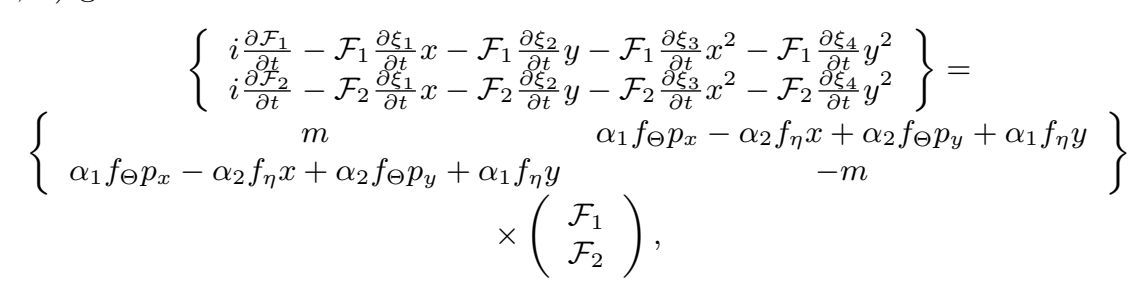

then, we obtain

$$
\begin{aligned}
& i \frac{\partial \mathcal{F}_{1}}{\partial t}-\mathcal{F}_{1} \frac{\partial \xi_{1}}{\partial t} x-\mathcal{F}_{1} \frac{\partial \xi_{2}}{\partial t} y-\mathcal{F}_{1} \frac{\partial \xi_{3}}{\partial t} x^{2}-\mathcal{F}_{1} \frac{\partial \xi_{4}}{\partial t} y^{2}=f_{\Theta} \mathcal{F}_{2} p_{x}+i f_{\eta} \mathcal{F}_{2} x-i f_{\Theta} \mathcal{F}_{2} p_{y}+f_{\eta} \mathcal{F}_{2} y+m \mathcal{F}_{1} \\
& i \frac{\partial \mathcal{F}_{2}}{\partial t}-\mathcal{F}_{2} \frac{\partial \xi_{1}}{\partial t} x-\mathcal{F}_{2} \frac{\partial \xi_{2}}{\partial t} y-\mathcal{F}_{2} \frac{\partial \xi_{3}}{\partial t} x^{2}-\mathcal{F}_{2} \frac{\partial \xi_{4}}{\partial t} y^{2}=f_{\Theta} \mathcal{F}_{1} p_{x}-i f_{\eta} \mathcal{F}_{1} x+i f_{\Theta} \mathcal{F}_{1} p_{y}+f_{\eta} \mathcal{F}_{1} y-m \mathcal{F}_{2}
\end{aligned},
$$

by solving the above system of equations, we find

$$
\begin{gathered}
\frac{\partial \mathcal{F}_{1}}{\partial t}=-i m \mathcal{F}_{1}, \quad \frac{\partial \mathcal{F}_{2}}{\partial t}=i m \mathcal{F}_{2} \\
\mathcal{F}_{1} \frac{\partial \xi_{1}}{\partial t}=-i\left\{\frac{e B}{2}+\frac{\eta}{2} e^{-\gamma t}\right\} \mathcal{F}_{2} \\
\mathcal{F}_{1} \frac{\partial \xi_{2}}{\partial t}=-\left\{\frac{e B}{2}+\frac{\eta}{2} e^{-\gamma t}\right\} \mathcal{F}_{2} \\
\frac{\partial \xi_{3}}{\partial t}=\frac{\partial \xi_{4}}{\partial t}=0
\end{gathered}
$$

which leads to obtaining

$$
\begin{gathered}
\mathcal{F}_{1}=e^{-i m t+q_{1}}, \quad \mathcal{F}_{2}=e^{i m t+q_{2}}, \\
\frac{\partial \xi_{1}}{\partial t}=i \frac{\partial \xi_{2}}{\partial t}=-i\left\{\frac{e B}{2}+\frac{\eta}{2} e^{-\gamma t}\right\} e^{i 2 m t+q_{2}-q_{1}}, \\
\xi_{1}=i \xi_{2}=-i\left\{\frac{\kappa}{4 l_{B}^{2} i m} e^{i 2 m t}+\frac{\eta \kappa}{4 i m-2 \gamma} e^{(-\gamma+i 2 m) t}\right\},
\end{gathered}
$$

with $q_{1}, q_{2}$ and $\kappa=e^{q_{2}-q_{1}}$ being real constants, $l_{B}^{-1}=\sqrt{e B}$ is the magnetic length [58]. In commutative case $(\Theta=\eta=\gamma=0)$, then the above relations 79 , 81 return to that of general quantum mechanics

$$
\xi_{1}(t)=i \xi_{2}(t)=-\frac{\kappa}{4 l_{B}^{2} m} e^{i 2 m t}, \quad|\phi(x, y, t\rangle|_{\eta=\gamma=0} \sim e^{-\frac{i \kappa}{4 l_{B}^{2} m} e^{i 2 m t}(x+i y)+o_{1} i x^{2}+o_{2} i y^{2}},
$$

with $o_{1}, o_{2}$ are real constants, and in $t=0$

$$
\xi_{1}(t=0)=i \xi_{2}(t=0)=-\frac{\kappa}{4 l_{B}^{2} m}, \quad \text { and } \mathcal{F}_{1}=\kappa \mathcal{F}_{2}=e^{q_{1}} .
$$

\section{Conclusion}

In conclusion, the dynamics of the system of a time-dependent NC Dirac equation has been analysed and formulated using the LR invariant method. We introduced the time-dependent noncommutativity using a timedependent Bopp-shift translation. Knowing that the NC structure constants postulated expanding exponentially with the evolution of time, and the time-dependency have a multitude of other possibilities.

We benefit from the dynamical invariant following the standard procedure allowed to construct and to obtain an analytical solution of the system. Having obtained the explicit solutions could also help to investigate and reformulate the modified version of Heisenberg's uncertainty relations emerging from non-vanishing commutation relations (1). The uncertainty for the observables A, B has to satisfy the inequality $\left.\triangle A \triangle B\right|_{\psi} \geq \frac{1}{2}|\langle\psi|[A, B]| \psi\rangle|$ with $\left.\triangle A\right|_{\psi} ^{2}=\left\langle\psi\left|A^{2}\right| \psi\right\rangle-\langle\psi|A| \psi\rangle^{2}$ and the same for B for any state. Depending on these results, we are planning to study the pair creation process, and investigate its implications in quantum optics. 


\section{ACKNOWLEDGEMENTS}

The author wishes to express thanks to Pr Lyazid Chetouani for his interesting comments and suggestions, and would also like to appreciate anonymous reviewers for their careful reading of the manuscript and their insightful comments.

\section{REFERENCES}

[1] H. S. Snyder. Quantized space-time. Physical Review 71:38 - 41, 1947. DOI:10.1103/PhysRev.71.38

[2] H. S. Snyder. The electromagnetic field in quantized space-time. Physical Review 72:68 - 71, 1947. DOI:10.1103/PhysRev.72.68.

[3] A. Connes, M. Douglas, A. Schwarz. Noncommutative geometry and matrix theory: Compactification on tori. Journal of High Energy Physics 1998, 1998.

[4] T. Banks, W. Fischler, S. H. Shenker, L. Susskind. $m$ theory as a matrix model: A conjecture. Physical Review D 55:5112 - 128, 1997. DOI:10.1103/PhysRevD.55.5112.

[5] M. Chaichian, M. M. Sheikh-Jabbari, A. Tureanu. Hydrogen atom spectrum and the lamb shift in noncommutative QED. Physical Review Letters 86:2716 - 2719, 2001. DOI:10.1103/PhysRevLett.86.2716.

[6] B. Mirza, M. Zarei. Non-commutative quantum mechanics and the Aharonov-Casher effect. European Physical Journal C 32:583 - 586, 2004. DOI:10.1140/epjc/s2003-01522-8.

[7] C. Duval, P. HorvÃ ithy. The exotic Galilei group and the "Peierls substitution". Physics Letters B 479(1):284 290, 2000. DOI:10.1016/S0370-2693(00)00341-5

[8] A. Connes. Non-commutative differential geometry. Publications Mathématiques de l'Institut des Hautes Études Scientifiques 62:41 - 144, 1985. DOI:10.1007/BF02698807.

[9] A. Connes. A short survey of noncommutative geometry. Journal of Mathematical Physics 41(6):3832 - 3866, 2000. DOI:10.1063/1.533329

[10] S. Woronowicz. Twisted SU (2) group. An example of a non commutative differential calculus. Publications of the Research Institute for Mathematical Sciences 23:117, 1987. DOI:10.2977/prims/1195176848

[11] N. Seiberg, E. Witten. String theory and noncommutative geometry. Journal of High Energy Physics 1999(09):032 - 032, 1999. DOI:10.1088/1126-6708/1999/09/032.

[12] J. Madore, S. Schraml, P. Schupp, J. Wess. Gauge theory on noncommutative spaces. The European Physical Journal C - Particles and Fields 16:161 - 167, 2000. DOI:10.1007/s100520050012.

[13] I. Haouam. Continuity equation in presence of a non-local potential in non-commutative phase-space. Open Journal of Microphysics 09:15 - 28, 2019. DOI:10.4236/ojm.2019.93003.

[14] I. Haouam. On the Fisk-Tait equation for spin-3/2 fermions interacting with an external magnetic field in noncommutative space-time. Journal of Physical Studies 24, 2020. DOI:10.30970/jps.24.1801

[15] I. Haouam. The non-relativistic limit of the DKP equation in non-commutative phase-space. Symmetry 11:223, 2019. DOI:10.3390/sym11020223

[16] I. Haouam, L. Chetouani. The Foldy-Wouthuysen transformation of the dirac equation in noncommutative phase-space. Journal of Modern Physics 09:2021 - 2034, 2018. DOI:10.4236/jmp.2018.911127

[17] B. Jurčo, L. Möller, S. Schraml, et al. Construction of non-Abelian gauge theories on noncommutative spaces. European Physical Journal C 21:383, 2001. DOI:10.1007/s100520100731

[18] J.-i. Kamoshita. Probing noncommutative space-time in the laboratory frame. The European Physical Journal $C$ 52:451, 2002. DOI:10.1140/epjc/s10052-007-0371-y

[19] Z. Alisultanov. Landau levels in graphene in crossed magnetic and electric fields: Quasi-classical approach. Physica B: Condensed Matter 438:41 - 44, 2014. DOI:10.1016/j.physb.2013.12.033

[20] Z. Alisultanov. Oscillations of magnetization in graphene in crossed magnetic and electric fields. JETP Letters 99:232 - 236, 2014. DOI:10.1134/S0021364014040055.

[21] N. Ma, S. Zhang, D. Liu, V. Wang. Influence of electrostatic field on the weiss oscillations in graphene. Physics Letters A 378(45):3354 - 3359, 2014. DOI:10.1016/j.physleta.2014.09.026.

[22] S. Zhang, N. Ma, E. Zhang. The modulation of the de Haas-van Alphen effect in graphene by electric field. Journal of Physics: Condensed Matter 22(11):115302, 2010. DOI:10.1088/0953-8984/22/11/115302.

[23] N. M. R. Peres, E. V. Castro. Algebraic solution of a graphene layer in transverse electric and perpendicular magnetic fields. Journal of Physics: Condensed Matter 19(40):406231, 2007. DOI:10.1088/0953-8984/19/40/406231

[24] K. Novoselov, A. Geim, S. Morozov, et al. Two-dimensional gas of massless dirac fermions in graphene. Nature 438:197 - 200, 2005. DOI:10.1038/nature04233.

[25] A. De Martino, L. Dell'Anna, R. Egger. Magnetic confinement of massless dirac fermions in graphene. Physical Review Letters 98:066802, 2007. DOI:10.1103/PhysRevLett.98.066802 
[26] L. Dell'Anna, A. De Martino. Multiple magnetic barriers in graphene. Physical Review B 79:045420, 2009. DOI:10.1103/PhysRevB.79.045420.

[27] Y. Zhang, Y.-W. Tan, H. Stormer, P. Kim. Experimental observation of the quantum Hall effect and Berry's phase in graphene. Nature 438:201 - 204, 2005. DOI:10.1038/nature04235.

[28] K. Bolotin, F. Ghahari, M. Shulman, et al. Observation of the fractional quantum Hall effect in graphene. Nature 462:196 - 199, 2009. DOI:10.1038/nature08582.

[29] G. P. Mikitik, Y. V. Sharlai. The Berry phase in graphene and graphite multilayers. Low Temperature Physics 34(10):794 - 800, 2008. DOI:10.1063/1.2981389.

[30] V. Lukose, R. Shankar, G. Baskaran. Novel electric field effects on landau levels in graphene. Physical Review Letters 98:116802, 2007. DOI:10.1103/PhysRevLett.98.116802.

[31] V. Arjona, E. V. Castro, M. A. H. Vozmediano. Collapse of landau levels in Weyl semimetals. Physical Review B 96:081110, 2017. DOI:10.1103/PhysRevB.96.081110.

[32] G. Burmeister, K. Maschke. Scattering by time-periodic potentials in one dimension and its influence on electronic transport. Physical Review B 57:13050 - 13060, 1998. DOI:10.1103/PhysRevB.57.13050.

[33] C. S. Tang, C. S. Chu. Coherent quantum transport in narrow constrictions in the presence of a finite-range longitudinally polarized time-dependent field. Physical Review B 60:1830 - 1836, 1999. DOI:10.1103/PhysRevB.60.1830.

[34] W. Li, L. E. Reichl. Transport in strongly driven heterostructures and bound-state-induced dynamic resonances. Physical Review B 62:8269 - 8275, 2000. DOI:10.1103/PhysRevB.62.8269.

[35] C. Figueira de Morisson Faria, M. Dörr, W. Sandner. Time profile of harmonic generation. Physical Review A 55:3961 - 3963, 1997. DOI:10.1103/PhysRevA.55.3961

[36] I. H. Deutsch, P. S. Jessen. Quantum-state control in optical lattices. Physical Review A 57:1972 - $1986,1998$. DOI:10.1103/PhysRevA.57.1972.

[37] H. Maeda, T. F. Gallagher. Nondispersing wave packets. Physical Review Letters 92:133004, 2004. DOI:10.1103/PhysRevLett.92.133004.

[38] C. E. Creffield, G. Platero. Ac-driven localization in a two-electron quantum dot molecule. Physical Review B 65:113304, 2002. DOI:10.1103/PhysRevB.65.113304.

[39] H. P. Yuen. Two-photon coherent states of the radiation field. Physical Review A 13:2226 - 2243, 1976. DOI:10.1103/PhysRevA.13.2226

[40] M. Governale, F. Taddei, R. Fazio. Pumping spin with electrical fields. Physical Review B 68:155324, 2003. DOI:10.1103/PhysRevB.68.155324.

[41] A. G. Mal'shukov, C. S. Tang, C. S. Chu, K. A. Chao. Spin-current generation and detection in the presence of an ac gate. Physical Review B 68:233307, 2003. DOI:10.1103/PhysRevB.68.233307

[42] L. S. Brown. Quantum motion in a Paul trap. Physical Review Letters 66:527 - 529, 1991. DOI:10.1103/PhysRevLett.66.527

[43] M. Feng. Complete solution of the Schrödinger equation for the time-dependent linear potential. Physical Review A 64:034101, 2001. DOI:10.1103/PhysRevA.64.034101

[44] M.-L. Liang, Z.-G. Zhang, K.-S. Zhong. Quantum-classical correspondence of the time-dependent linear potential. Czechoslovak Journal of Physics 54:397 - 402, 2004. DOI:10.1023/B:CJOP.0000020579.42018.d9.

[45] H. R. Lewis. Classical and quantum systems with time-dependent harmonic-oscillator-type hamiltonians. Physical Review Letters 18:510 - 512, 1967. DOI:10.1103/PhysRevLett.18.510

[46] H. R. Lewis, W. B. Riesenfeld. An exact quantum theory of the time-dependent harmonic oscillator and of a charged particle in a time-dependent electromagnetic field. Journal of Mathematical Physics 10(8):1458 - 1473, 1969. DOI:10.1063/1.1664991.

[47] I. A. Pedrosa, J. L. Melo, E. Nogueira. Linear invariants and the quantum dynamics of a nonstationary mesoscopic RLC circuit with a source. Modern Physics Letters B 28(27):1450212, 2014. DOI:10.1142/S0217984914502121.

[48] X. Chen, A. Ruschhaupt, S. Schmidt, et al. Fast optimal frictionless atom cooling in harmonic traps: Shortcut to adiabaticity. Physical Review Letters 104:063002, 2010. DOI:10.1103/PhysRevLett.104.063002.

[49] S. Dey, A. Fring. Noncommutative quantum mechanics in a time-dependent background. Physical Review D 90:084005, 2014. DOI:10.1103/PhysRevD.90.084005.

[50] V. V. Nesvizhevsky, H. G. Börner, A. M. Gagarski, et al. Measurement of quantum states of neutrons in the Earth's gravitational field. Physical Review D 67:102002, 2003. DOI:10.1103/PhysRevD.67.102002

[51] V. Nesvizhevsky, H. BÃ $₫$ rner, A. Petukhov, et al. Quantum states of neutrons in the Earth's gravitational field. Nature 415:297 - 299, 2002. DOI:10.1038/415297a. 
[52] S. M. Carroll, J. A. Harvey, V. A. Kostelecký, et al. Noncommutative field theory and Lorentz violation. Physical Review Letters 87:141601, 2001. DOI:10.1103/PhysRevLett.87.141601

[53] O. Bertolami, J. G. Rosa, C. M. L. de Aragão, et al. Noncommutative gravitational quantum well. Physical Review D 72:025010, 2005. DOI:10.1103/PhysRevD.72.025010

[54] F. Delduc, Q. Duret, F. Gieres, M. Lefrancois. Magnetic fields in noncommutative quantum mechanics. Journal of Physics: Conference Series 103:012020, 2008. DOI:10.1088/1742-6596/103/1/012020

[55] X. Jiang, C. Long, S. Qin. Solution of dirac equation with the time-dependent linear potential in non-commutative phase space. Journal of Modern Physics 04:940 - 944, 2013. DOI:10.4236/jmp.2013.47126

[56] H. Sobhani, H. Hassanabadi. Two-dimensional linear dependencies on the coordinate time-dependent interaction in relativistic non-commutative phase space. Communications in Theoretical Physics 64(3):263 - 268, 2015. DOI:10.1088/0253-6102/64/3/263.

[57] M. Merad, S. Bensaid. Wave functions for a Duffin-Kemmer-Petiau particle in a time-dependent potential. Journal of Mathematical Physics 48(7):073515, 2007. DOI:10.1063/1.2747609.

[58] Z. Jiang, E. A. Henriksen, L. C. Tung, et al. Infrared spectroscopy of landau levels of graphene. Physical Review Letters 98:197403, 2007. DOI:10.1103/PhysRevLett.98.197403 